\title{
Monitoring of lipodystrophic and metabolic abnormalities in HIV-1 infected children on antiretroviral therapy
}

\author{
Vana Spoulou, ${ }^{1}$ Christina Kanaka-Gantenbein, ${ }^{1}$ Irini Bathrellou, ${ }^{2}$ Stefano Mora, ${ }^{3}$ \\ Glyceria Mostrou, ${ }^{1}$ Lambros Sidossis, ${ }^{2}$ George Chrousos, ${ }^{1}$ Maria Theodoridou ${ }^{1}$
}

${ }^{1}$ First Department of Pediatrics, University of Athens, “Agia Sophia” Children's Hospital, Athens, ${ }^{2}$ Laboratory of Nutrition and Clinical Dietetics, Department of Nutrition and Dietetics, Harokopio University, Athens, Greece, ${ }^{3}$ Laboratory of Pediatric Endocrinology, Division of Metabolic and Cardiovascular Sciences, San Raffaele Scientific Institute, Milan, Italy

\begin{abstract}
OBJECTIVE: Few studies have thus far assessed body composition by dual energy X-ray absorptrometry (DXA) in children with HIV, primarily because reference data for normally growing children and adolescents are not available. Our study aimed at evaluating body composition in children with HIV and their relation to serum lipids and glucose homeostasis. DESIGN: Body composition was assessed by DXA in $17 \mathrm{HIV}-1$ infected children at entrance to the study and after 12 months and in one hundred fifty nine age, gender, body mass index (BMI), and Tanner stage matched healthy subjects who served as controls. Lipodystrophy was diagnosed if the trunk/leg fat ratio was out of the range of the expected mean \pm 1SD of the controls. RESULTS: At study entry, 10 patients ( 7 girls) had developed lipohypertrophy, whereas all remaining patients had lipoatrophy. Lipohypertrophy was associated with older age $(p=0.027)$. Lipodystrophic phenotype was stable in all patients except in one over the 12-month period of follow-up while on continuous antiretroviral therapy (ART). 80\% and $70 \%$ of patients with lipohypertrophy had triglycerides and cholesterol levels, respectively above the $7^{\text {th }}$ percentile, while $57 \%$ and $43 \%$ of patients with lipoatrophy had triglycerides and cholesterol, respectively greater than the $75^{\text {th }}$ percentile. High triglycerides were associated with the use of protease inhibitors $(p=0.028)$. Basal fasting glucose and homeostasis model assessment (HOMA) values were within normal limits. CONCLUSIONS: HIV-infected paediatric patients on continuous ART developed significant and persistent body composition changes which were associated with dyslipidemia without overt abnormalities of glucose metabolism.
\end{abstract}

Key words: Antiretroviral therapy, Children, DXA, Dyslipidemia, HIV, Lipodystrophy

Address for correspondence:

Vana Spoulou, “Agia Sophia” Children's Hospital, Infectious

Diseases Dept, Thivon and M. Asias, Goudi 11527 ,

e-mail: vspoulou@med.uoa.gr, Tel.: +30 6944149266,

Fax: +302107467663

Received 10-09-10, Revised 01-02-11, Accepted 30-02-11

\section{INTRODUCTION}

Lipodystrophy (LD) syndrome, characterized by altered fat distribution and lipid and glucose metabolism abnormalities, namely dyslipidemia and insulin resistance, is quite common among HIV-infected adults on antiretroviral therapy (ART) and is ac- 
companied by an increased risk of atherosclerotic cardiovascular morbidity and mortality. ${ }^{1,2}$

In paediatric patients on ART, changes in body composition manifested as central fat accumulation, lipoatrophy or a combination of these, as well as abnormalities in lipid and glucose metabolism, do occur but there is controversy regarding their incidence, severity and associated atherosclerosis risk factors. ${ }^{3,4}$ The onset of fat redistribution in children is usually subtle and studies based on clinical criteria have low sensitivity for early detection of such changes. ${ }^{5}$ Dual energy X-ray absorptiometry (DXA) is considered the gold standard for the objective evaluation of body composition. ${ }^{6}$ However, in children the interpretation of DXA data is difficult because body composition shows a great variation with age, gender and pubertal status and there are no reference data for normally growing children and adolescents. Therefore, only a few studies have used DXA to objectively identify altered fat distribution in children with HIV., ${ }^{7,8}$ Furthermore, studies on the possible association of lipodystrophic phenotype with metabolic changes have yielded conflicting results. ${ }^{9-11}$

Our aim was therefore to evaluate body composition by DXA in a cohort of Greek HIV-1 infected children and adolescents on ART. The data were compared with those observed in a large group of strictly age, gender, body mass index (BMI), and Tanner stage-matched healthy children who had no clinical signs or predisposing factors for lipodystrophy. Moreover, in our HIV children we related lipodystrophic phenotype to serum lipids and glucose homeostasis at entrance to the study and 12 months later, while the subjects were on continuous exposure to ART.

\section{Study population}

Seventeen children vertically infected with HIV-1 and followed at "Aghia Sophia" Children's Hospital, Athens, Greece, were included in the study. All patients were on ART with at least 3 drugs for a period of $5.2 \pm 4.76$ (mean \pm DS) years. At study entry, 6 children were prepubertal and 11 were pubertal [Stage II (2), III (2), IV (3) V (4)], while during the following 12 months one prepubertal child entered puberty [Stage II (2), III (2), IV (3), V (5)]. As a control group for DXA data, we studied 159 Caucasian subjects whose ages ranged from 4-21 years and who had no history of endocrine, nutritional, growth or renal problems and had no signs of LD. Their height, weight and BMI values were between the $3^{\text {rd }}$ and $97^{\text {th }}$ centile for age and gender.

The study received ethical approval by the hospital ethics committee and written informed consent was obtained from the parents or guardians of all study subjects before inclusion in the study.

\section{METHODS}

All patients were assessed by the same physician for body weight and height, pubertal status according to Tanner stage and clinical characteristics of LD, e.g. wasting in the extremities, face or buttocks and/ or fat accumulation in the abdomen or dorsocervical spine (buffalo hump).

Soft tissue composition of the whole body and of regions such as arms, legs and trunk were determined by DXA (model DPX-MD+, Lunar Corp., Madison, WI, USA). A fast scan mode was used with a scanning time of approximately $15 \mathrm{~min}$. Total body scans were analyzed by Lunar software (version 4.7e).

Total cholesterol, high (HDL) and low (LDL) density lipoprotein cholesterol, triglycerides, plasma glucose and serum insulin concentrations were measured in the $17 \mathrm{HIV}-1$ children by standard methodology in blood samples taken after overnight fasting. Insulin resistance was calculated according to the homeostasis model assessment (HOMA) ${ }^{12}$

\section{STATISTICAL ANALYSIS}

Expected body composition values were obtained separately for boys and girls by regression analyses on controls' data. Second and $3^{\text {rd }}$ degree regression best fitted the body composition variables plotted against age. Patients' measurements were compared with expected values by paired t-test. In HIV-infected subjects with different lipodystrophic phenotypes, analysis of covariance (ANCOVA) was used to evaluate the differences in biochemical indices over the 12-month period and after adjusting for age. Logistic regression was used for the computation of odds ratios. Fisher's exact tests were used for comparison of proportions, and Student's t test for the comparison of means and evaluation of possible differences at baseline and 
follow-up measurements. Generalized estimating equations assessed changes in categorically defined outcomes between the two time periods. Chi-square tests were used for the comparison of proportions. $\mathrm{P}$ values reported are two-tailed. Statistical significance was set at 0.05 and analysis was conducted using STATA 7.0.

\section{RESULTS}

\section{Study subjects}

Seventeen HIV-infected children (10 girls, 7 boys) aged (mean $\pm \mathrm{SD}$ ) $12.5 \pm 3.98$ (range: $5-17.5$ ) years, weight SDS $-0.344 \pm 0.96$, height SDS $-0.425 \pm 1.26$ and BMI z score $-0.227 \pm 1.13$. The mean (range) CD4 cell count and viral load (by reverse-transcriptase-PCR, Amplicor Roche, Alameda, USA) were 876.3 (145.4 -1858 ) cells $/ \mathrm{ml}$ and $1.05 \times 10^{3}$ (range $<50-1.3 \times 10^{5}$ ) vc/ $\mathrm{ml}$, respectively. At entrance to the study, all patients had been receiving ART with at least 3 drugs for a (mean $\pm \mathrm{SD}$ ) period of $5.2 \pm 4.76$ years; $64.7 \%$ were receiving at least one protease inhibitor (PI). During the 12-month follow-up period, one patient had to change his treatment regimen based on genotype resistance pattern and by the end of the study, 58.8\% of the children were on at least one PI. At the end of the follow-up period, mean CD4 counts and viral load were 953.9 cells $/ \mathrm{ml}$ and $1.01 \times 10^{3} \mathrm{vc} / \mathrm{ml}$, respectively.

The 159 controls ( 79 girls, 84 boys) included in the study had the following characteristics: 75 girls and 84 boys with a mean age of $12.7(\mathrm{SD}=4.9$, range: 4-21.9) years, weight SDS $-0.2 \pm 1.1 \mathrm{~kg}$, height SDS $-0.1 \pm 1.2 \mathrm{~m}$ and BMI SDS $-0.2 \pm 1.1 \mathrm{~kg} / \mathrm{m}^{2}$.

\section{Body composition measurements}

There were no significant differences in body composition detected by DXA in HIV-infected children compared to controls. However, fat distribution expressed as trunk/leg fat ratio was significantly higher in patients compared to the expected values derived from the controls, indicating central fat accumulation [(1.03 \pm 0.30 vs $0.81 \pm 0.09), \mathrm{p}=0.003]$ (Table 1$)$.

To further investigate body changes, we considered trunk/leg fat ratio greater than the mean value in the control group $+1 \mathrm{SD}$ as indicating lipohypertrophy (central fat accumulation), and a ratio less than the mean values of the controls as indicating lipoatrophy.
Table 1. Values of various parameters of body composition in HIV infected patients (n: 17) at study entry and in controls (expected values)

\begin{tabular}{lccc}
\hline Variable & $\begin{array}{c}\text { Observed } \\
\text { Mean (SD) }\end{array}$ & $\begin{array}{c}\text { Expected* } \\
\text { Mean (SD) }\end{array}$ & $\mathbf{p}$ \\
\hline Trunk fat $(\mathrm{kg})$ & $4.4(3.0)$ & $3.8(1.9)$ & 0.20 \\
Legs fat $(\mathrm{kg})$ & $4.0(2.3)$ & $4.5(1.9)$ & 0.10 \\
Tibia lean $(\mathrm{kg})$ & $29.2(10.8)$ & $28.0(9.3)$ & 0.28 \\
Legs lean $(\mathrm{kg})$ & $9.8(4.4)$ & $9.8(3.5)$ & 0.91 \\
Arms lean $(\mathrm{kg})$ & $2.9(1.5)$ & $2.6(0.9)$ & 0.04 \\
Trunk fat/legs fat & $1.03(0.3)$ & $0.81(0.09)$ & 0.0037 \\
BMI z score & $-0.227(1.13)$ & $-0,2(1.1)$ & 0.87 \\
\hline
\end{tabular}

*Expected values were obtained separately for boys and girls, by regression analysis on controls' data.

Based on this definition, at study entry 10 pubertal patients (7 girls) had lipohypertrophy. The remaining patients (4 boys, 3 girls) had lipoatrophy with significantly less total and regional fat compared to the controls (Table 2). When DXA-based diagnosis of LD was compared with clinically diagnosed fat distribution, it was found that central fat accumulation expressed as waist circumference above the $75^{\text {th }}$ centile for their gender and age ${ }^{13}$ was present in only 5 of the 10 patients diagnosed by DXA as having lipohypertrophy.

The mean (SD) age of patients with lipohypertrophy was 13.9 (2.9) years and 9.2 (4) years in patients with lipoatrophy $(\mathrm{OR}=1.4,95 \% \mathrm{CI}: 1.12-1.97$ $\mathrm{p}=0.027$ ), suggesting a significant association between older age and the development of lipohypertrophic LD. Although there was an association between increased duration of treatment and lipohypertrophy differences, this did not reach significance $(\mathrm{OR}=1.64$, 95\% CI: $0.93-1.88, \mathrm{p}=0.075)$. Moreover, $72.7 \%$ of subjects with lipohypertrophy were receiving ART with at least $1 \mathrm{PI}$; however, differences between subjects with lipohypertrophy compared to those with lipoatrophy did not reach statistical significance $(p=0.162)$. Female gender was more frequent among subjects with lipohypertrophy, $70 \%$ vs $42 \%$ in subjects with lipoatrophy, although differences did not reach significance. Stage of HIV, CD4 counts and viral load were not associated with LD phenotype ( $p>0.05)$.

Lipodystrophic phenotype did not change after 1 year of additional exposure to ART in all patients 


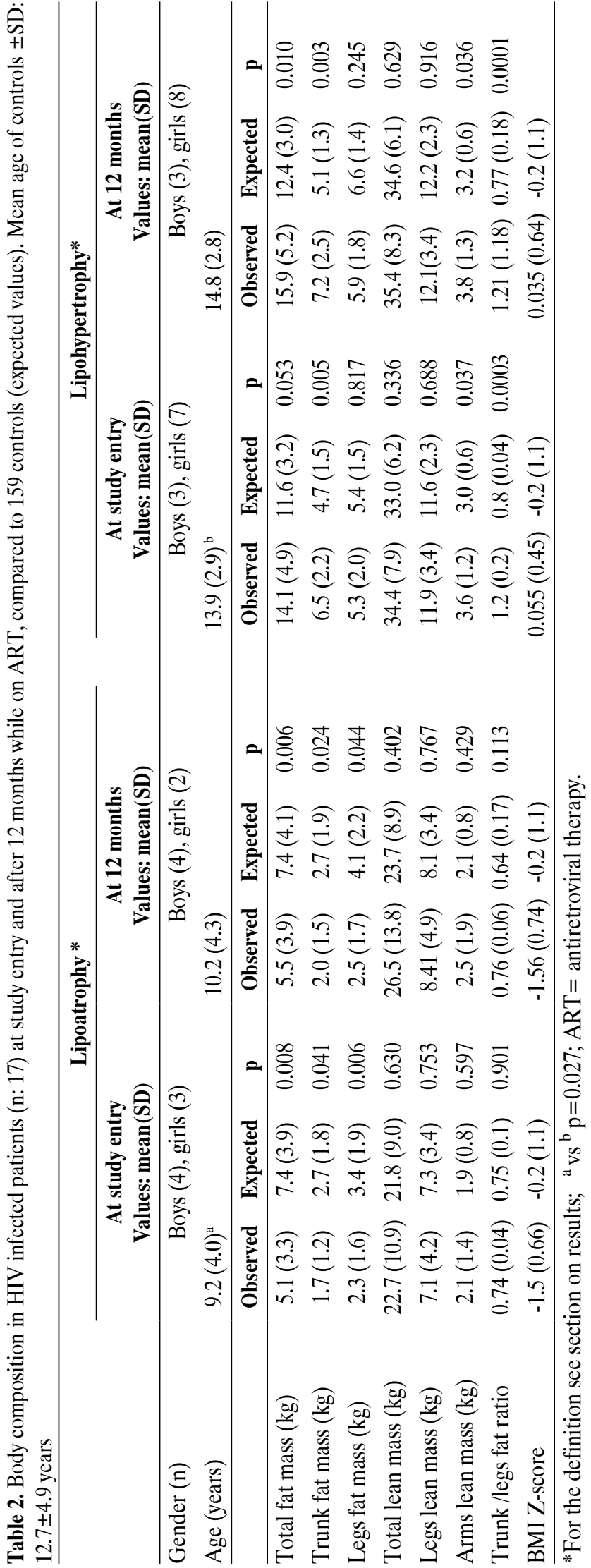

except one prepubertal girl with lipoatrophy who entered puberty and developed central fat accumulation. In all patients, there was a non-statistically significant increase in total and regional fat and lean mass at 12 months. Differences in the degree of change between study entry and at 12 months for each variable were not significant between subjects with lipoatrophy and those with lipohypertrophy (Table 2).

\section{Association of Lipodystrophic phenotype with metabolic abnormalities}

At study entry, $80 \%$ and $70 \%$ of patients with lipohypertrophy had triglycerides and cholesterol levels above the $75^{\text {th }}$ percentile for their age, respectively, compared to $57 \%$ and $43 \%$ of patients with lipoatrophy $(p=0.04)$. The calculation was based on cholesterol and triglyceride distribution data of 7767 school-aged Greek children. ${ }^{14}$ Baseline mean (SD) triglycerides and cholesterol concentrations were 1.789 (1.423) mmol/L and 4.929 (1.194) mmol/L, respectively. Higher triglycerides were significantly associated with the use of PIs (OR=20.0, 95\% CI: 1.39-287.60, $\mathrm{p}=0.028)$. Patients on longer duration of ART had triglycerides more frequently above the $75^{\text {th }}$ percentile. However, the association did not reach statistical significance $(\mathrm{OR}=4.01,95 \% \mathrm{CI}$ : 0.80-20.02, $\mathrm{p}=0.091$ ).

At 12 months, all subjects with lipohypertrophy had triglycerides above the $75^{\text {th }}$ percentile and mean values were higher compared to subjects with lipoatrophy $(p=0.068)$. In contrast, cholesterol levels remained stable and there were no differences between the two groups; HDL (mean, SD) and LDL (mean, SD) cholesterol concentrations were normal at baseline and after 12 months in all studied subjects; $1.29(0.31)$ vs $1.27(0.36)$ and $2.88(1.12)$ vs $2.88(0.78) \mathrm{mmol} / \mathrm{L}$ for HDL and LDL at baseline and 12 months, respectively.

There were no differences between data at study entry and 12 months later for fasting glucose, fasting insulin and HOMA either in subjects with lipohypertrophy or in those with lipoatrophy (Table 3 ).

\section{DISCUSSION}

A DXA-based assessment was used to determine body composition in HIV-1 infected paediatric patients on ART and the possible association of lipodys- 
Table 3. Metabolic paremeters in HIV-infected patients according to lipodystrophic phenotype at entrance to the study and 12 months later

\begin{tabular}{|c|c|c|c|c|c|c|c|}
\hline & \multicolumn{2}{|c|}{$\begin{array}{c}\text { Values } \\
\text { at study entry }\end{array}$} & \multicolumn{2}{|c|}{$\begin{array}{c}\text { Values } \\
\text { at } 12 \text { months }\end{array}$} & \multicolumn{2}{|c|}{ Change } & \multirow[b]{2}{*}{$\mathbf{P} \dagger \mathbf{P} \doteqdot$} \\
\hline & Mean & $\mathrm{SE}$ & Mean & $\mathrm{SE}$ & Mean & SE & \\
\hline \multicolumn{8}{|c|}{ Triglycerides (mmol/L) } \\
\hline LA & 1.25 & 0.28 & 0.74 & 0.12 & -0.51 & 0.21 & 0.1120 .643 \\
\hline $\mathrm{LH}$ & 2.14 & 0.57 & 1.45 & 0.26 & -0.69 & 0.48 & 0.208 \\
\hline $\mathrm{P}^{*}$ & 0.249 & & 0.029 & & & & \\
\hline \multicolumn{8}{|c|}{ Cholesterol (mmol/L) } \\
\hline LA & 5.00 & 0.47 & 4.63 & 0.48 & -0.37 & 0.36 & 0.5210 .774 \\
\hline $\mathrm{LH}$ & 4.88 & 0.41 & 4.51 & 0.49 & -0.38 & 0.34 & 0.600 \\
\hline $\mathrm{P}^{*}$ & 0.861 & & 0.858 & & & & \\
\hline \multicolumn{8}{|c|}{ Fasting glucose $(\mathrm{mmol} / \mathrm{L})$} \\
\hline LA & 4.62 & 0.16 & 4.47 & 0.07 & -0.15 & 0.14 & $0.325 \quad 0.820$ \\
\hline $\mathrm{LH}$ & 4.67 & 0.17 & 4.47 & 0.07 & -0.21 & 0.18 & 0.276 \\
\hline $\mathrm{P}^{*}$ & 0.837 & & 0.969 & & & & \\
\hline \multicolumn{8}{|c|}{ Insulin $(\mathrm{pmol} / \mathrm{L})$} \\
\hline LA & 32.53 & 7.85 & 22.58 & 9.56 & -9.95 & 12.37 & $0.654 \quad 0.713$ \\
\hline $\mathrm{LH}$ & 53.93 & 8.64 & 32.07 & 7.20 & -21.86 & 10.73 & 0.109 \\
\hline $\mathrm{P}^{*}$ & 0.096 & & 0.445 & & & & \\
\hline \multicolumn{8}{|c|}{ HOMA } \\
\hline LA & 1.06 & 0.28 & 0.57 & 0.24 & -0.49 & 0.35 & $0.700 \quad 0.945$ \\
\hline LH & 1.58 & 0.31 & 0.90 & 0.21 & -0.68 & 0.42 & 0.136 \\
\hline $\mathrm{P}^{*}$ & 0.760 & & 0.310 & & & & \\
\hline
\end{tabular}

* $p$ value for group (lipoatrophy vs lipohypertrophy) effect; $\dagger p$ value for time effect; $\uparrow p$ value for differences in the degree of change for each variable between the two groups; LA: Lipoatrophic phenotype, LH: Lipohypertrophic phenotype.

trophic phenotype with biochemical abnormalities at study entry and 12 months later while on continuous ART. We identified deviations in fat distribution in all seropositive subjects using optimal cut-off values generated by comparison of patients' body composition values with those of a large group of strictly age, sex, BMI and Tanner stage-matched controls. The strength of our study was the assessment of LD by DXA, which is an objective and highly sensitive method for early detection of body composition abnormalities. ${ }^{6}$ We were able to interpret the results of DXA by carefully selecting a large group of matched controls, which enabled us to calculate accurately the normally expected body composition values and objectively define their body composition deviations.

Although the lack of a control group consisting of patients not receiving ART is a weakness of our study, our findings suggest that all HIV-infected patients after some years on ART develop LD changes pre- senting either as lipohypertrophy or as lipoatrophy. Interestingly, in our study central fat accumulation was clinically evident in only half of the patients with lipohypertrophy, suggesting that the clinical diagnosis of lipodystrophy is not always possible. ${ }^{7,15}$

In accordance with previous reports, we demonstrated that lipohypertrophy with central fat accumulation was more frequent among patients with longer exposure to ART and advanced pubertal development, whereas lipoatrophy was more common in younger children. ${ }^{7,16}$ The relative contribution of puberty and duration of ART cannot be assessed. Most subjects with lipohypertrophy were on PIs; however, differences between the two groups were not significant.

LD phenotype was stable in all but one patient in the 12 months' evaluation. In contrast to adults, who lose subcutaneous fat at a rate of $12-15 \%$ per year, ${ }^{17}$ all our patients had an increase in fat and lean mass over the 12-month period, a phenomenon at- 
tributed to their good nutrition and disease control. Subjects with lipohypertrophy retained higher total and trunk fat mass, whereas patients with lipoatrophy, total and regional fat remained significantly lower than expected. The only patient with progressive LD changes during the follow-up period was a girl with lipoatrophy who entered puberty and developed central fat accumulation. We demonstrated a worse lipohypertrophic phenotype among pubertal HIVinfected females, an observation pointing to a gender effect on the development of metabolic abnormalities and body adipose tissue distribution possibly through an effect of estrogens. It is known that estrogens' action during puberty facilitates lipid accumulation, as is the case in healthy adolescent girls, in contrast to the action of testosterone in males, where muscle accruement is the rule. In line with this, it has been reported that female gender is a predisposing factor for fat accumulation in other studies of HIV-infected adolescents. ${ }^{7,18}$

Most of our patients with lipohypertrophy had dyslipidemia mainly manifested as triglycerides values above the $75^{\text {th }}$ centile for their age. Similarly to previous reports, the use of PIs was significantly associated with the development of higher triglycerides. ${ }^{5}$ The underlying mechanism associating the development of LD and dyslipidemia with antiviral compounds could be their effect on hepatic lipid synthesis and metabolism. The prevailing hypothesis regarding the development of lipoatrophy is that NRTI-mediated mitochondrial dysfunction in adipocytes leads to fat cell apoptosis and depletion. ${ }^{18}$ The association of central fat accumulation and dyslipidemia with the use of PIs has been attributed to their inhibiting activity on HIV-1 aspartic acid protease, which is a protein sharing a $60 \%$ homology with low density lipoprotein receptor. It has been suggested that by changing serum lipid concentrations and increasing rates of apoptosis in peripheral adipocytes might lead to visceral abdominal adipose tissue accumulation since fewer peripheral adipocytes would be available to process circulating fatty acids. Other important mechanisms are those associated with dysregulation of cytokines such as TNFa, IL-1 $\beta$ and IL- 6 caused by the HIV infection per se and/or expression of the HIV-1 encoding proteins such as Vpr and Tat. These proteins change the activities of glucocotricoid and
PPAR receptors and may also be implicated in the expression of HIV-related metabolic syndrome. ${ }^{19}$

Insulin resistance is considered a basic pathogenetic factor of the LD syndrome observed in adult HIV-infected patients. ${ }^{2}$ We could not demonstrate any overt impairment of glucose metabolism nor insulin resistance based on serum fasting glucose and insulin levels. HOMA an insulin resistance index, was higher among subjects with lipohypertrophy; however, our study was not powered enough to detect significant differences and the follow-up period was relatively short. Previous small studies in pediatric HIV-infected patients have also shown that a great percentage of lipodystrophic body changes were observed without any evidence of insulin resistance. ${ }^{20}$ However, Bitnun et al, using the more sensitive method of the frequent sampling during an i.v. glucose tolerance test, reported an impaired $\beta$ cell response in PI-treated children in a manner similar to that observed in adults. ${ }^{21}$

The long-term impact of LD on atherosclerotic risk of HIV-infected children is uncertain. Plebani et al studied seropositive children for 5 years and reported absence of cardiovascular events related to metabolic abnormalities. ${ }^{22}$ However, the cumulative effects of ART over time, especially for children starting therapy at a very young age, is still unknown. It has however been shown that lipohypertrophy is directly associated with increased visceral fat storage which is linked to an increased atherosclerotic risk. ${ }^{7}$ Children with lipohypertrophic phenotype detected by DXA could be further assessed with MRI, which is considered the gold standard for an accurate estimation of visceral adiposity in paediatric patients, but less convenient and not widely available. The early identification of patients with central fat accumulation and dyslipidemia, which have been clearly shown to increase the risk of cardiovascular disease, could help to appropriately modify treatment, e.g. replacing PI with non-nucleotide reverse transcriptase analogs that have a beneficial effect on dyslipidemia as well as lifestyle interventions. ${ }^{17}$

In conclusion, our study conducted in, an admittedly, small group of patients, demonstrated that HIV-infected paediatric patients on continuous ART develop significant and persistent deviations in body composition which are frequently associated with 
dyslipidemia. The assessment of body composition in association with the close monitoring of serum lipids and glucose homeostasis could help in the early identification of subjects who require modification of ART or other measures in order to improve their prognosis.

\section{ACKNOWLEDGMENTS}

We are grateful to Ms H. Tzavara MSc, who performed the statistical analysis.

\section{No conflict of interest or financial support declared}

\section{REFERENCES}

1. Grinspoon S, Carr A, 2005 Cardiovascular risk and body-fat abnormalities in $\mathrm{HIV}$-infected adults. N Engl J Med 352: 48-62.

2. Leow MK, Addy CL, Mantzoros CS, 2003 Clinical review 159: Human immunodeficiency virus/highly active antiretroviral therapy-associated metabolic syndrome: clinical presentation, pathophysiology, and therapeutic strategies. J Clin Endocrinol Metab 88: 1961-1976.

3. Jaquet D, Levine M, Ortega-Rodriguez E, et al, 2000 Clinical and metabolic presentation of the lipodystrophic syndrome in HIV-infected children. AIDS 14: 2123 2128.

4. Amaya RA, Kozinetz CA, McMeans A, Schwarzwald H, Kline MW, 2002 Lipodystrophy syndrome in human immunodeficiency virus-infected children. Pediatr Infect Dis J 21: 405-410.

5. Taylor P, Worrell C, Steinberg SM, et al, 2004 Natural history of lipid abnormalities and fat redistribution among human immunodeficiency virus-infected children receiving long-term, protease inhibitor-containing, highly active antiretroviral therapy regimens. Pediatrics 114: e235-242.

6. Ellis KJ, Shypailo RJ, Pratt JA, Pond WG, 1994 Accuracy of dual-energy x-ray absorptiometry for bodycomposition measurements in children. Am J Clin Nutr 60: 660-665.

7. Vigano A, Mora S, Testolin C, et al, 2003 Increased lipodystrophy is associated with increased exposure to highly active antiretroviral therapy in HIV-infected children. J Acquir Immune Defic Syndr 32: 482-489.

8. Brambilla P, Bricalli D, Sala N, et al, 2001 Highly active antiretroviral-treated HIV-infected children show fat distribution changes even in absence of lipodystrophy. AIDS 15: 2415-2422.

9. Aurpibul L, Puthanakit T, Lee B, Mangklabruks A, Sirisanthana T, Sirisanthana V, 2007 Lipodystrophy and metabolic changes in HIV-infected children on non-nucleoside reverse transcriptase inhibitor-based antiretroviral therapy. Antivir Ther 12: 1247-1254.

10. Krause JC, Toye MP, Fisher DJ, Stechenberg BW, Reiter EO, Allen HF, 2009 Metabolic abnormalities in human immunodeficiency virus-infected children: two-year follow-up. J Pediatr Endocrinol Metab 22: 345-351.

11. Parakh A, Dubey AP, Kumar A, Maheshwari A, 2009 Lipodystrophy and metabolic complications of highly active antiretroviral therapy. Indian J Pediatr 76: 10171021.

12. Matthews DR, Hosker JP, Rudenski AS, Naylor BA, Treacher DF, Turner RC, 1985 Homeostasis model assessment: insulin resistance and beta-cell function from fasting plasma glucose and insulin concentrations in man. Diabetologia 28: 412-419.

13. Fernandez J, Redden D, Pietrobelli A, Allison D, 2004 Waist circumference percentiles innationally representative sampes of African-American,European-American and Mexican-American children and adolescents. J Pediatr 145: 439-444.

14 Schulpis K, Karikas GA, 1998 Serum cholesterol and triglyceride distribution in 7767 school-aged Greek children. Pediatrics 101: 861-864.

15. European Pediatric Lipodystrophy Group, 2004 Antiretroviral therapy, fat redistribution and hyperlipidaemia in HIV-infected children in Europe. AIDS 18: 1443-1451.

16. Sanchez Torres AM, Munoz Muniz R, Madero R, Borque C, Garcia-Miguel MJ, De Jose Gomez MI, 2005 Prevalence of fat redistribution and metabolic disorders in human immunodeficiency virus-infected children. Eur J Pediatr 164: 271-276.

17. Haubrich RH, Riddler SA, DiRienzo AG, et al, 2009 Metabolic outcomes in a randomized trial of nucleoside, nonnucleoside and protease inhibitor-sparing regimens for initial HIV treatment. AIDS 23: 1109-1118.

18. Beregszaszi M, Dollfus C, Levine M, et al, 2005 Longitudinal evaluation and risk factors of lipodystrophy and associated metabolic changes in HIV-infected children. J Acquir Immune Defic Syndr 40: 161-168.

19. Kino T, Chrousos GP, 2003 AIDS-related insulin resistance and lipodystrophy syndrome. Curr Drug Targets Immune Endocr Metabol Disord 3: 111-117.

20. Bitnun A, Sochett E, Babyn P, et al, 2003 Serum lipids, glucose homeostasis and abdominal adipose tissue distribution in protease inhibitor-treated and naive HIV-infected children. AIDS 17: 1319-1327.

21. Bitnun A, Sochett E, Dick PT, et al, 2005 Insulin sensitivity and beta-cell function in protease inhibitor-treated and -naive human immunodeficiency virus-infected children. J Clin Endocrinol Metab 90: 168-174.

22. Plebani A, Esposito S, Pinzani R, et al, 2004 Effect of highly active antiretroviral therapy on cardiovascular involvement in children with human immunodeficiency virus infection. Pediatr Infect Dis J 23: 559-563. 